\title{
ON THE STABILITY OF THE FUNCTIONAL EQUATION
}

$$
\begin{gathered}
\phi[f(x)]=g(x) \phi(x)+F(x) \\
\text { E. TURDZA }
\end{gathered}
$$

ABSTRACT. This paper extends the results of D. Brydak [1] on the stability of the functional equation in the title.

1. This paper is concerned with the stability (defined below) of the functional equation

$$
\phi[f(x)]=g(x) \phi(x)+F(x) \quad(a<x<b)
$$

where $\phi$ is unknown and $f, g, F$ are given functions about which the following blanket hypotheses are made:

H1. $f, g, F \in C[a, b] ; g(x) \neq 0(a \leqq x \leqq b)$.

H2. $f(x)$ is strictly increasing and for some $\xi, a \leqq \xi \leqq b$,

$$
\begin{array}{ll}
x<f(x)<\xi & (a<x<\xi), \\
\xi<f(x)<x & (\xi<x<b) .
\end{array}
$$

REMARK. The values $a=-\infty, b=\infty$ need not be excluded, but if $a=-\infty$ we must assume that $f, g, F$ tend to limits as $x \rightarrow-\infty$. Similarly for $b=\infty$.

By the hypotheses $\mathrm{H} 1$ and $\mathrm{H} 2$,

$$
f(\xi)=\xi \text { and } f([a, b]) \subset[a, b] .
$$

In this paper $f^{n}$ denotes the composition product $f \circ f \circ \cdots \circ f, n$ times. $f^{-1}$ is the inverse function of $f, f^{-n}=\left(f^{-1}\right)^{n}$.

Following Brydak [1] we define stability as follows.

Let $S \psi(x)=g\left[f^{-1}(x)\right] \psi\left[f^{-1}(x)\right]+F\left(f^{-1}(x)\right)$ for all $x$ belonging to a subinterval $I$ of $[a, b] . S$ is a transformation $S: C(I) \rightarrow C(f(I))$.

It is easily verified that

$$
S^{n} \psi(x)=\prod_{j=1}^{n} g\left[f^{-j}(x)\right] \cdot \psi\left(f^{-n}(x)\right)+h_{n}(x),
$$

where $h_{n}$ is independent of $\psi$. If $\phi$ is a solution of (1) in $I$, then

$$
S \phi[f(x)]=\phi[f(x)] \quad(x \in I)
$$

Received by the editors October 3, 1969 and, in revised form, February 9, 1971. AMS 1970 subject classifications. Primary 39A15.

Key words and phrases. Functional equation. 
and so, by iteration,

$$
S^{n} \phi\left[f^{n}(x)\right]=\phi\left[f^{n}(x)\right] \quad(x \in I) .
$$

The equation (1) is called stable, if there is a $K>0$ such that for every $\psi \in C(I)$ which satisfies

$$
\left\|\psi\left(f^{n}(x)\right)-S^{n} \psi\left(f^{n}(x)\right)\right\|<\epsilon \quad(n=1,2, \cdots)
$$

there is a solution $\phi$ of (1) such that

$$
\|\psi(x)-\phi(x)\|<K \epsilon \quad(x \in I) .
$$

Here $\|\cdots\|$ is the $C(I)$ norm.

2. D. Brydak [1] gave conditions under which (1) is stable in an interval $\left[\xi, x_{0}\right]\left(x_{0}<b\right)$.

We add here

ThEOREM 1. The equation (1) is stable in every interval $\left[x_{0}, b\right)$ $\left(\xi<x_{0}<b\right)$, if either

(a) for some integer $r, f^{r}(b)<x_{0}$, or

(b) $g(x) \geqq 1$ in a neighborhood $\left(x_{1}, b\right]$ of $b$.

We need the following lemma, proved in [2].

Lemma. Let $\xi<x_{0}<b$. Then, by $\mathrm{H} 2$,

$$
I_{0}=\left[f\left(x_{0}\right), x_{0}\right] \subset[\xi, b] \text {. }
$$

Let $\phi_{0}(x) \in C\left(I_{0}\right), \phi_{0}\left(f\left(x_{0}\right)\right)=S \phi_{0}\left(f\left(x_{0}\right)\right)$ and $\prod_{i=0}^{n-1} g\left[f^{i}(x)\right]$ tends to zero uniformly on $I_{0}$.

Then there exists a continuous solution $\phi$ of $(1)$ in $(\xi, b)$ such that

$$
\phi(x)=\phi_{0}(x) \quad\left(x \in I_{0}\right) .
$$

Proof of Theorem 1. Let $\psi \in C\left[f\left(x_{0}\right), b\right]$ and let $\psi$ satisfy (3) where the norm is the supremum norm in $\left[f\left(x_{0}\right), b\right]$. Let $\eta(x)$ be a linear function which decreases from 1 to 0 as $x$ increases from $f\left(x_{0}\right)$ to $x_{0}$. Put

$$
\phi_{0}(x)=\psi(x)+\eta(x)\left\{S \psi\left(f\left(x_{0}\right)\right)-\psi\left(f\left(x_{0}\right)\right)\right\}
$$

By (3),

$$
\left|\phi_{0}(x)-\psi(x)\right|<\epsilon \quad\left(x \in I_{0}\right)
$$

and, by the definition of $S$,

$$
\begin{aligned}
S \phi_{0}\left(f\left(x_{0}\right)\right) & =g\left(x_{0}\right) \phi_{0}\left(x_{0}\right)+F\left(x_{0}\right)=g\left(x_{0}\right) \psi\left(x_{0}\right)+F\left(x_{0}\right) \\
& =S \psi\left(f\left(x_{0}\right)\right)=\phi_{0}\left(f\left(x_{0}\right)\right) .
\end{aligned}
$$


Applying the Lemma with this choice of $\phi_{0}$ we obtain a solution $\phi$ of $(1)$ in $(\xi, b)$. This solution satisfies, for any $x \in(\xi, b)$ and any natural integer,

$$
\begin{aligned}
\mid \phi\left(f^{k}(x)\right) & -\psi\left(f^{k}(x)\right) \mid \\
= & \left|S^{k} \phi\left(f^{k}(x)\right)-\psi\left(f^{k}(x)\right)\right| \\
\geqq & \left|S^{k} \phi\left(f^{k}(x)\right)-S^{k} \psi\left(f^{k}(x)\right)\right|-\left|\psi\left(f^{k}(x)\right)-S^{k} \psi\left(f^{k}(x)\right)\right| .
\end{aligned}
$$

For every $x \in\left[x_{0}, b\right)$, there is a unique natural integer $k$ such that $f^{k}(x) \in I_{0}$. (Otherwise, by $\mathrm{H} 2, f^{k}(x) \rightarrow X, x_{0} \leqq X$ as $k \rightarrow \infty$. But then $f(X)=X$, contradicting H2.)

Choosing $k$ such that $f^{k}(x) \in I_{0}$ we have, by (4), (5), (2) and (3),

$$
\begin{aligned}
\epsilon & >\left|\phi_{0}\left(f^{k}(x)\right)-\psi\left(f^{k}(x)\right)\right|=\left|\phi\left(f^{k}(x)\right)-\psi\left(f^{k}(x)\right)\right| \\
& >G_{k}(x)|\phi(x)-\psi(x)|-\epsilon,
\end{aligned}
$$

where $G_{k}(x)=\prod_{j=0}^{k-1} g\left(f^{j}(x)\right)$.

The theorem follows from (6), if we can prove the existence of a positive constant $m$ such that

$$
\left|G_{k}(x)\right| \geqq m>0 \quad\left(k=1,2, \cdots ; x \in\left[x_{0}, b\right]\right) .
$$

In case (a), $k \leqq r$ and we can choose

$$
m=\inf _{0 \leqq k \leqq r} \inf _{x_{0} \leqq x \leqq b}\left|G_{k}(x)\right| .
$$

In case (b), let $M$ be the integer such that $f^{M}\left(x_{1}\right) \in I_{0}$. For any $x$ in $\left[x_{0}, x_{1}\right]$ we can use the argument of case (a) with $r=M$. For $x_{1}<x<b$ there is an index $l$ such that $y=f^{l}(x)<x_{1} \leqq f^{l-1}(x)$. By case (a) and (6) with $k=l$ there is a constant $m$ such that

$$
\begin{aligned}
2 \epsilon / m & >|\phi(y)-\psi(y)|=\left|\phi\left(f^{l}(x)\right)-\psi\left(f^{l}(x)\right)\right| \\
& >G_{l}(x)|\phi(x)-\psi(x)|-\epsilon>|\phi(x)-\psi(x)|-\epsilon,
\end{aligned}
$$

since every factor in $G_{l}(x)$ is $\geqq 1$ by hypothesis.

\section{REFERENCES}

1. D. Brydak, On the stability of the linear functional equation $\phi[f(x)]$ $=g(x) \phi(x)+F(x)$, Proc. Amer. Math Soc. 26 (1970), 455-460.

2. M. Kuczma, Functional equations in a single variable, Monografie Mat., Tom 46, PWN, Warsaw, 1968. MR 37 \#4441.

Kraków, Ul. Idzikowskiego 19/4, Poland 